\title{
Probing primordial statistical anisotropy with WMAP and Planck data
}

\author{
Sabir Ramazanov* \\ Universite Libre de Bruxelles \\ E-mail: Sabir.Ramazanov@ulb.ac.be
}

\begin{abstract}
We constrain several models of the early Universe that predict statistical anisotropy of the cosmic microwave background (CMB) sky. We make use of WMAP9 and Planck maps deconvolved with beam asymmetries. As compared to previous releases of CMB data, they do not exhibit the anomalously large quadrupole of statistical anisotropy. This allows to strengthen the limits on the parameters of models established earlier in the literature. Among the others, we discuss constraints on the class of models with the Maxwellian term non-minimally coupled to the inflaton and a row of alternatives to inflation, i.e., Galilean genesis and conformal rolling scenario. The talk is based on the results obtained in the papers $[1,2,3]$.
\end{abstract}

References

[1] S.R. Ramazanov and G. I. Rubtsov, Statistical anisotropy of CMB as a probe of conformal rolling scenario, JCAP 1205 (2012) 033, arXiv:1202.4357 [astro-ph.CO].

[2] S. R. Ramazanov and G. I. Rubtsov, Constraining anisotropic models of the early Universe with WMAP9 data, Phys. Rev. D 89 (2014) 043517, arXiv:1311.3272 [astro-ph.CO].

[3] G. Rubtsov, S. Ramazanov, F. Urban, M. Thorsrud, Probing primordial statistical anisotropy with Planck data, work in progress.

Frontiers of Fundamental Physics 14 - FFP14,

15-18 July 2014

Aix Marseille University (AMU) Saint-Charles Campus, Marseille

\footnotetext{
*Speaker.
} 